\title{
Intramolecular cleavage of the hASRGL1 homodimer occurs in two stages
}

Wenzong $\mathrm{Li}^{1}$, Seema Irani ${ }^{2}$, Amanda Crutchfield ${ }^{1}$, Kristal Hodge ${ }^{1}$, Wendy Matthews ${ }^{1}$, Pooja Patel $^{1}$, Yan Jessie Zhang ${ }^{1,3}$ and Everett Stone ${ }^{1 *}$

${ }^{1}$ Department of Molecular Biosciences, ${ }^{2}$ Chemical Engineering, ${ }^{3}$ Institute of Cellular and Molecular Biology, University of Texas, Austin, Texas 78712.

*Address correspondence to:

Yan Jessie Zhang: 1 University Station A5300, Austin, TX 78712. Phone: (512)-471-8645. Fax: 512-471-9469. E-mail: jzhang@cm.utexas.edu \&

Everett Stone: 1 University Station C0800, Austin, TX 78712. Phone: (512) 512-232-4103.

E-mail: stonesci@utexas.edu

SUPPORTING INFORMATION 


\section{SUPPORTING FIGURES}

Figure S1. SDS-PAGE analysis of WT-hASRGL1 incubated with $1 \mathrm{M}$ glycine over time.

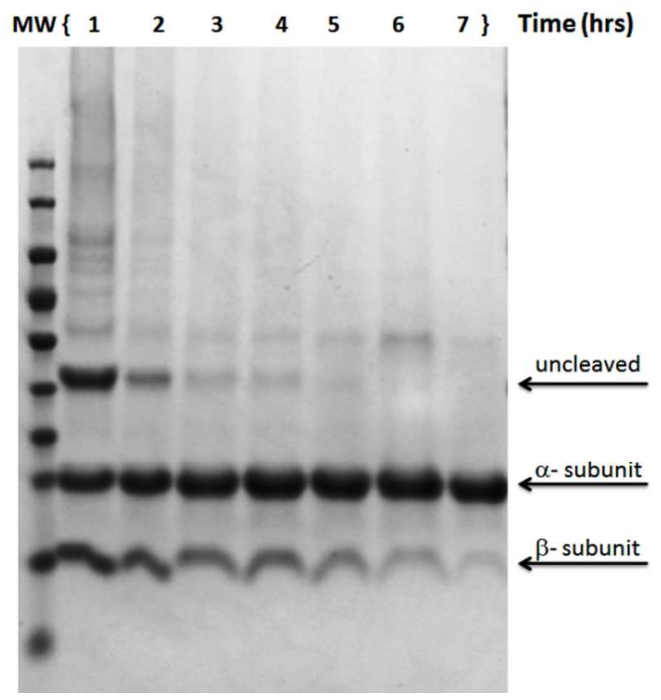


Figure S2. Size-exclusion chromatography profiles of WT-hASRGL1 and hASRGL1-C202 variants. WT-hASRGL1 (blue), hASRGL1C202V (purple), C202S (brown), and C202A (orange).

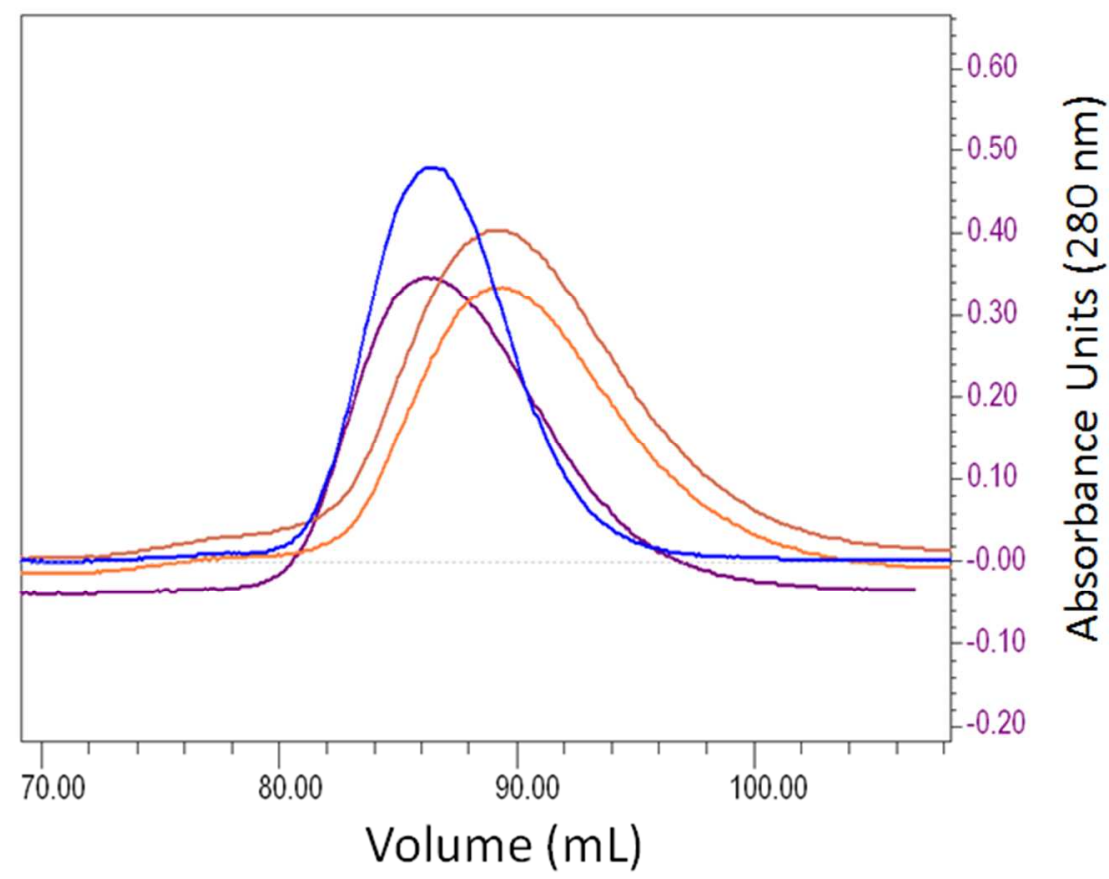


Figure S3. Composite SDS-PAGE gel of WT-hASRGL1 and hASRGL1-C202 variants. The top bands represents full length uncleaved protein. The two smaller bands represent $\alpha$ and $\beta$ domains after intramolecular processing (without glycine). After $48 \mathrm{hrs}$ incubation in $37^{\circ} \mathrm{C}, \sim 50 \%$ of wthASRGL1 is cleaved while the hASRGL1-C202A, hASRGL1-C202S, and hASRGL1-C202G have no detectable processing.

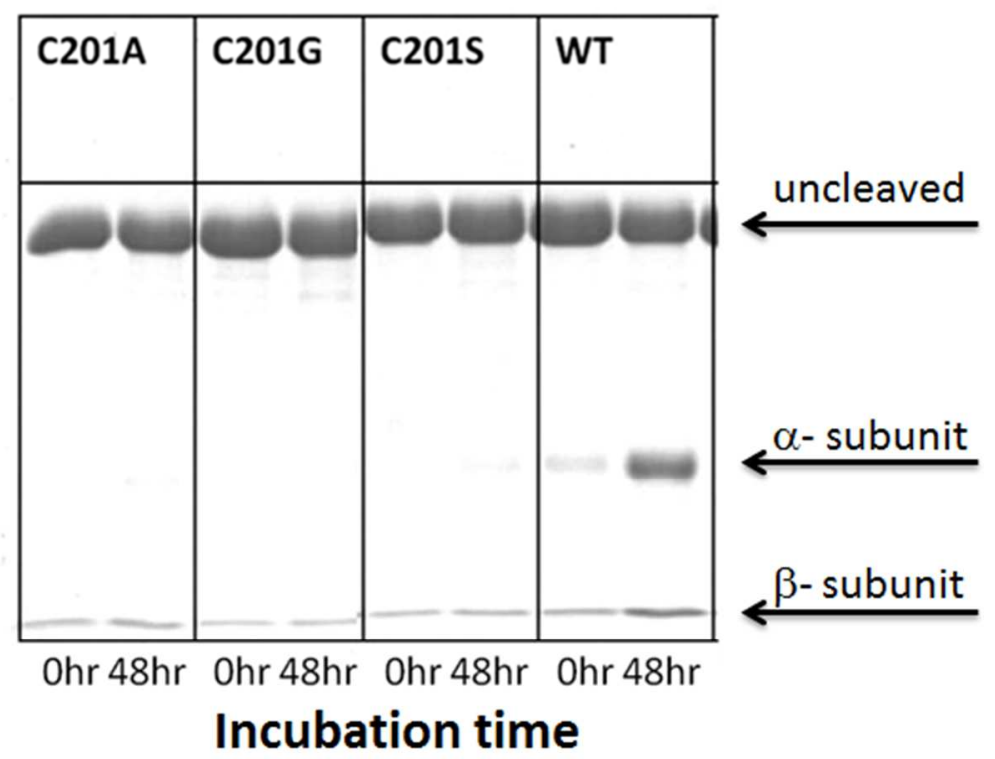




\section{SUPPORTING TABLES}

\section{Table S1}

Table S1. Thermal transition temperatures of hASRGL1 and variants $\left({ }^{\circ} \mathrm{C}\right)$

\begin{tabular}{lccccc} 
Glycine & - & - & + & + & + \\
\hline Oligomer & MM & MD & MM & MD & DD \\
WT & 61 & 65 & 71 & 79 & 86 \\
C201V & 59 & 62 & 70 & 79 & 86 \\
C201S & 49 & nd & 57 & 65 & 74 \\
C201 A & 49 & nd & 56 & 66 & 75 \\
\hline nd = not detected & & & & & \\
\hline
\end{tabular}




\section{Table S2}

Table S2. Thermal transition temperatures of hASRGL1 activated with small molecules $\left({ }^{\circ} \mathrm{C}\right)$ Oligomer $\quad$ MM MD

\begin{tabular}{llll}
\hline Glycine & 71 & 79 & 86 \\
Sarcosine & 65 & 72 & 80 \\
Phosphate & 66 & 73 & 78 \\
\hline
\end{tabular}

(1)

